\title{
La mesure du vent : conventions, instruments et données
}

The measurement of wind : conventions, instruments and data

Windmessung : Regeln, Instrumente, Daten

Pierre-Philippe Kastendeuch

\section{(2) OpenEdition}

Journals

Édition électronique

URL : http://journals.openedition.org/rge/4093

DOI : $10.4000 /$ rge.4093

ISSN : $2108-6478$

Éditeur

Association des géographes de l'Est

Édition imprimée

Date de publication : 1 juin 2000

ISSN : 0035-3213

\section{Référence électronique}

Pierre-Philippe Kastendeuch, «La mesure du vent : conventions, instruments et données », Revue Géographique de l'Est [En ligne], vol. 40 / 3 | 2000, mis en ligne le 02 août 2013, consulté le 08 septembre 2020. URL : http://journals.openedition.org/rge/4093 ; DOI : https://doi.org/10.4000/rge. 4093

Ce document a été généré automatiquement le 8 septembre 2020

Tous droits réservés 


\title{
La mesure du vent : conventions, instruments et données
}

\author{
The measurement of wind : conventions, instruments and data \\ Windmessung : Regeln, Instrumente, Daten
}

Pierre-Philippe Kastendeuch

\section{NOTE DE L'AUTEUR}

Remerciements à Daniel Viville (chercheur CNRS), Georges Najjar (maître de conférences) et Gilles Drogues (doctorant) qui m'ont gracieusement mis à disposition toutes les données nécessaires aux quelques graphiques présentés ici. Je tiens également à mentionner les firmes qui m'ont aimablement autorisé à publier les photos de capteurs, et notamment Campbell Scientific.

\section{Introduction}

1 Le vent est une force naturelle dont l'origine repose sur des mécanismes atmosphériques qui provoquent des différences de pression, ce qui met l'air en mouvement. Ces mouvements se traduisent par des déplacements plus ou moins rapides des gaz qui composent l'atmosphère et des particules qui s'y trouvent en suspension. Le vent est donc une variable atmosphérique tout à fait fondamentale à mesurer. En effet il conditionne, par exemple, la dispersion ou la concentration des polluants autour des sites industriels ou dans les villes. C'est également une variable qu'il faut connaître en agronomie, puisque le risque de gel au printemps peut être aggravé par temps calme, ce qui entraîne des dégâts importants sur certaines cultures sensibles. Des régions viticoles n'hésitent d'ailleurs pas à installer leur propre réseau météorologique de surveillance (Beltrando 1998). Dans un autre domaine, des pays comme le Danemark ont beaucoup misé sur le développement de l'énergie éolienne, puisque cette nation, par exemple, espère porter la part de la production énergétique 
éolienne à $20 \%$ d'ici 2008 (Laali et Benard, 1999). La force dégagée par le vent peut également être vécue comme une contrainte climatique majeure, capable d'occasionner des dégâts considérables aux infrastructures les plus solides, voire même d'affaiblir certains pans économiques d'un ou même de plusieurs pays simultanément. Les deux tempêtes qui se sont succédées sur les pays d'Europe de l'ouest en décembre 1999 en sont un bon exemple. Si ce risque est un fait assez exceptionnel dans nos régions, n'oublions pas que des secteurs des Etats-Unis sont touchés saisonnièrement par des tornades qui ravagent tout sur leur passage et que dans la zone intertropicale, d'autres pays sont soumis au passage récurrent de cyclones. L'énergie qui est mise en jeu dans ces phénomènes est colossale puisqu'un cyclone peut dégager de 200 à 300 kilotonnes par seconde (bombe d'Hiroshima: 20 kilotonnes). Loin de ces phénomènes extrêmement violents, le vent peut également être vécu comme une contrainte quasi quotidienne, à tel point qu'il a fallu en tenir compte dans l'aménagement du territoire : par exemple, les haies brise-vent des paysages de la vallée du Rhône, fréquemment sujette au mistral, et qui sont destinées à protéger les cultures (mais aussi les habitations et leurs occupants), de ce vent constant et pénible à supporter (Guyot, 1997). Compte tenu de toutes ces implications, il n'est pas étonnant de constater que toute une série de capteurs ont été mis au point pour mieux comprendre la circulation du vent et les mécanismes qui lui donnent naissance.

\section{Les réseaux de mesures}

2 Pour comprendre les phénomènes météorologiques et pour les prévoir, l'atmosphère est soumise à une surveillance constante par des réseaux de mesures qui doivent être les plus denses possibles, étant donné la grande variabilité spatiale des phénomènes. Ainsi en figure 1 nous avons une vision de la densité du réseau synoptique mondial. L'ensemble des points indique les stations continentales et océaniques. On constate d'emblée une faible couverture océanique, et même au sein des continents, des régions entières sont ignorées, ce qui pose de graves problèmes. En Europe, par contre, la densité est maximale; en effet, nos pays sont quadrillés par des réseaux de mesures denses et complets, qui sont entretenus par les services météorologiques nationaux comme Météo-France. Mais parfois ces réseaux de base se révèlent insuffisants et doivent encore être complétés par des équipements mis en place pour des recherches spécifiques dans le domaine de l'agronomie (INRA), de l'hydrologie (EDF) ou de la climatologie (Universités). Tous ces réseaux de mesures sont donc conçus de manière à quadriller au mieux le territoire, selon un maillage précis : en général une station tous les $60 \mathrm{~km}$ pour le réseau synoptique français et moins pour les autres types de réseaux. 
Fig. 1 : Carte d'implantation des stations météorologiques du réseau synoptique mondial

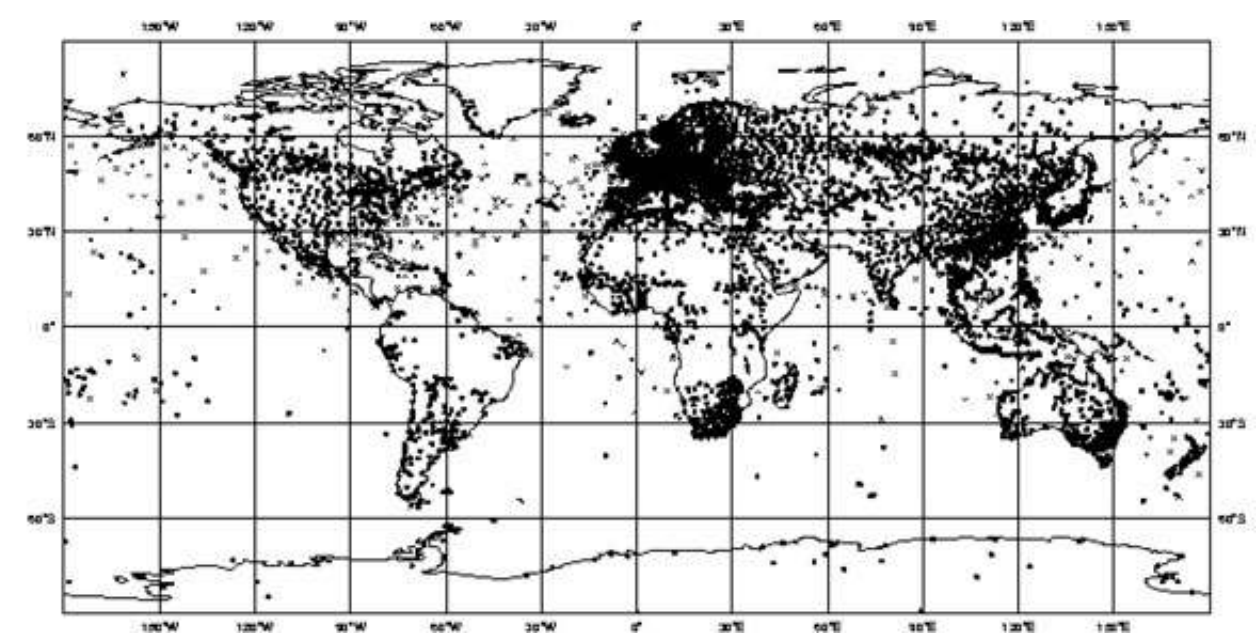

Chaque point représente une station.

Dans la grande majorité des cas, les stations météorologiques en fonctionnement sont aujourd'hui automatiques. Elles sont pilotées par des centrales d'acquisition d'une puissance comparable à celle d'ordinateurs personnels et qui sont capables d'interroger les différents types d'instruments, d'enregistrer les données sur une mémoire locale, de les traiter en temps réel et éventuellement les transmettre à distance, généralement par voie téléphonique (figure 2). Ce type de station automatique ne nécessite plus en principe la présence de personnel humain et effectue en routine une surveillance atmosphérique continue du territoire en mesurant les différents paramètres météorologiques fondamentaux : température, humidité, précipitation, vent et parfois pression, rayonnement... Cependant les stations synoptiques françaises (on en compte en général une par département) qui sont les mieux équipées et qui mesurent le plus de variables, sont toujours accompagnées de personnel en service permanent, chargé de superviser et de centraliser l'ensemble des mesures.

Fig. 2 : Exemple d'une station météorologique destinée à la recherche et munie d'une centrale d'acquisition de type CR10X

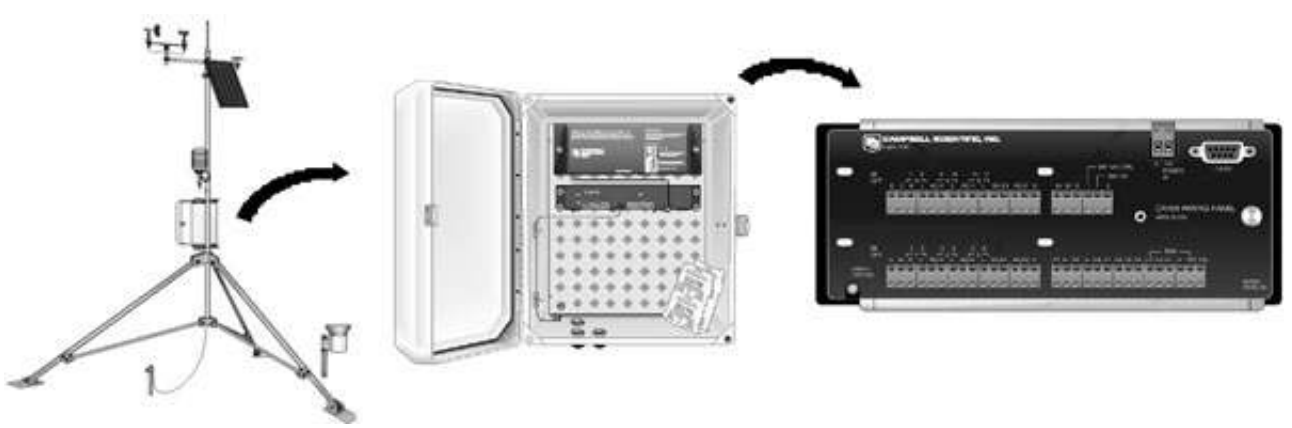

Matériel commercialisé par Campbell, photos Campbell Scientific. 


\section{Recommandations générales pour l'installation d'un poste d'observation}

4 Les variables météorologiques sont extrêmement sensibles aux conditions dans lesquelles elles sont mesurées (environnement du site de mesures, type de capteur...), ce qui explique pourquoi l'organisation météorologique mondiale (OMM, 1996) préconise un certain nombre de normes à respecter pour que les données puissent être comparées d'un poste à l'autre, et même entre pays. La surface sur laquelle doivent être installés les capteurs doit être, en principe, horizontale et couverte d'une végétation courte (en général de l'herbe régulièrement tondue). De plus, pour que les mesures soient représentatives du climat local, il faut que la végétation soit homogène dans un rayon d'au moins $100 \mathrm{~m}$ autour du site et qu'elle soit elle-même représentative des espèces locales.

5 Comme le vent est une variable particulièrement délicate à mesurer, le site doit être bien dégagé et se situer loin de tout bâtiment ou d'arbres, de façon à éliminer les effets thermodynamiques qu'ils induisent. En général on préconise de s'éloigner d'une distance égale à 10 fois la hauteur des obstacles les plus proches. De plus, la surface terrestre exerce une influence très importante sur les derniers centaines de mètres de l'atmosphère près $d u$ sol, de sorte que la vitesse du vent est considérablement freinée par la rugosité de ce dernier, ce qui entraîne une chute de vitesse par effet de cisaillement, qui, dans certains cas, peut se modéliser par une loi logarithmique ou exponentielle (figure 3). Donc pour procéder aux mesures, il faut s'éloigner au maximum du sol. La hauteur standard qui est recommandée est de $10 \mathrm{~m}$. Pour cette raison, la mesure du vent nécessite une infrastructure matérielle importante, puisque les capteurs doivent être hissés sur des mâts. Evidemment, certaines recherches poussées nécessitent des données à des niveaux intermédiaires (en agronomie par exemple), ce qui implique une multiplicité des niveaux de mesures de même style que sur la station météorologique présentée en figure 4. En outre, le vent étant extrêmement variable, il est très difficile d'extrapoler les données collectées en un endroit, ce qui pose également le problème de la représentativité des mesures. Pour palier cet inconvénient il faut multiplier fortement le nombre de postes pour espérer rendre compte des phénomènes qui se produisent sur un espace donné. A titre d'exemple on peut citer le nombre de postes installés pour une recherche destinée à mesurer la circulation du vent dans une vallée vosgienne en 1994 (Kastendeuch et Kaufmann, 1996; Kastendeuch et al. 2000). Dans cette vallée de $230 \mathrm{~km} 2$ environ, 14 postes de mesures du vent ont été installés (figure 5), soit l'équivalent des $2 / 3$ du réseau d'observation de toute la région Alsace. 
Fig. 3 : Vitesse du vent enregistrée au pylône de mesures du KFK à Karlsruhe (Allemagne) le 16 septembre 1992 à 1 heure du matin

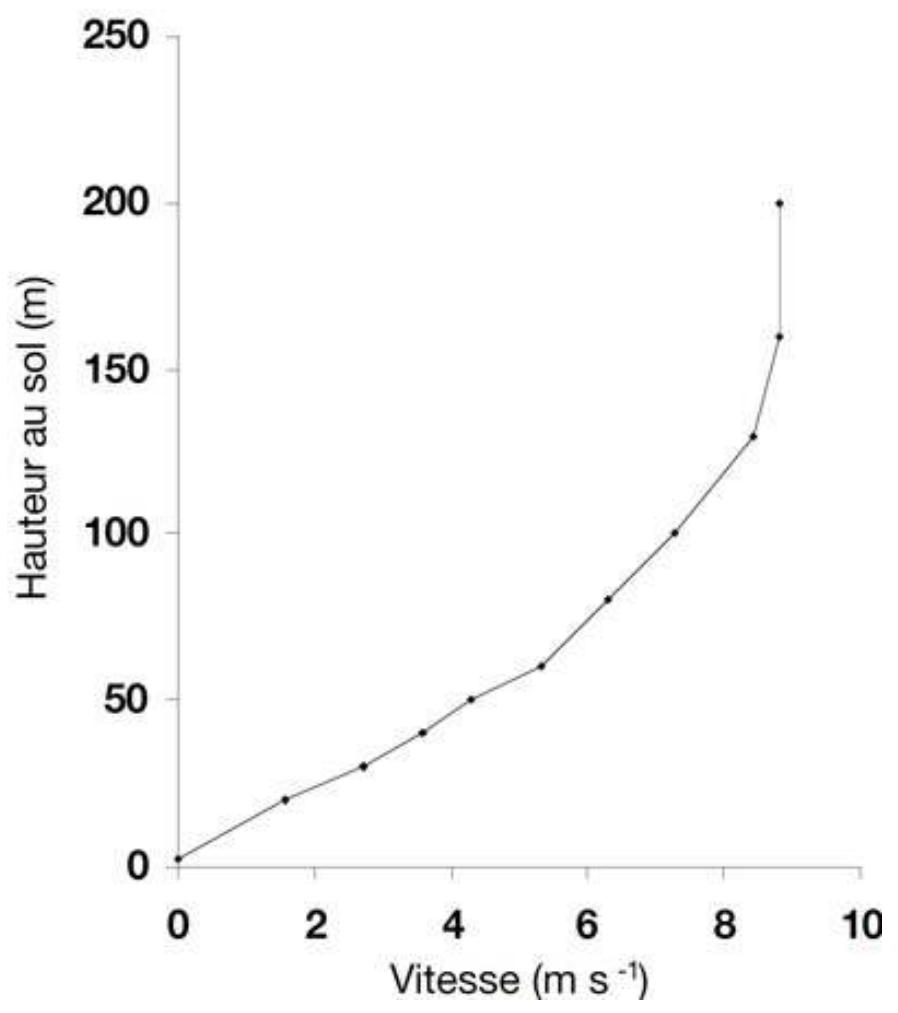

Données IMK 
Fig. 4 : Station météorologique de recherche de Lange Erlen (Suisse)

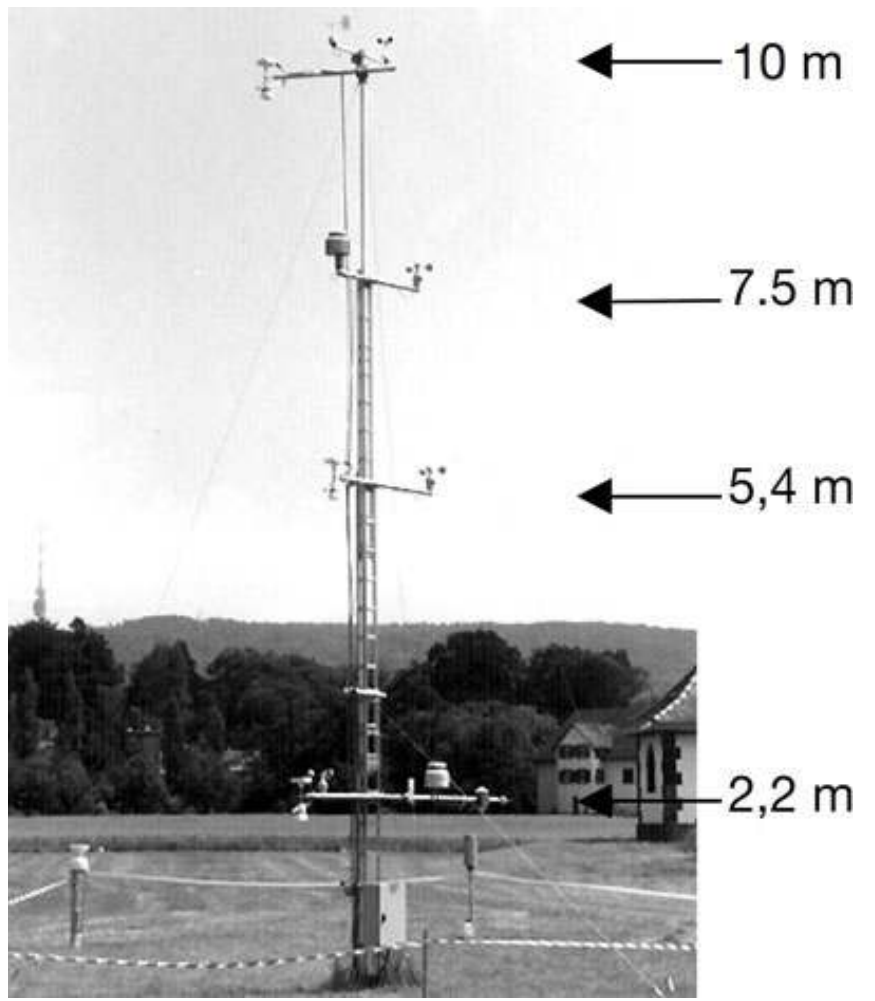

Les hauteurs de mesures du vent sont indiquées en mètres.

Photo reklip du Geographisches Institut der Universität Basel

Fig. 5 : Carte d'implantation des stations météorologiques installées dans la vallée de la Fecht (Alsace, France) en été 1994 par le Centre d'Études et de Recherches Éco-Géographiques.

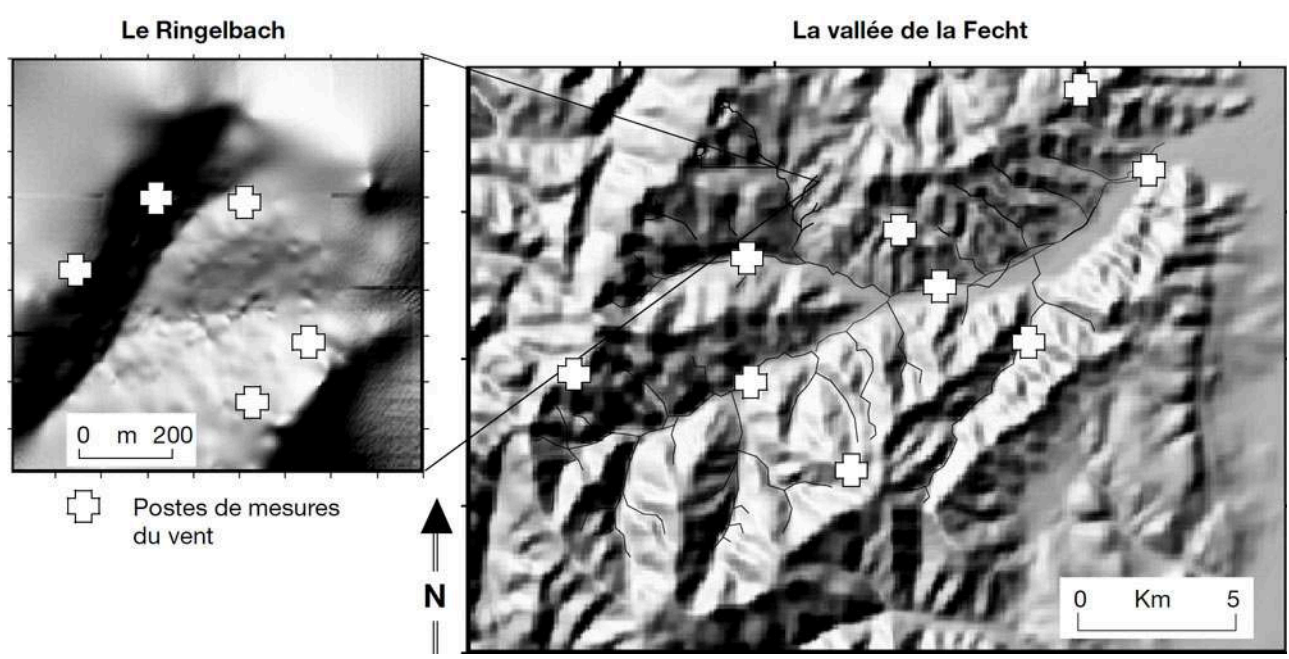

\section{Les capteurs}

Dans le domaine des capteurs destinés à mesurer le vent, on recherche surtout des instruments très sensibles (capables de ressentir les moindres fluctuations), qui possèdent un seuil de démarrage le plus bas possible, qui possèdent un minimum 
d'inertie et qui sont capables de résister aux vents les plus violents. C'est donc en fonction de ces critères, mais également en fonction d'objectifs précis de recherche, que sont régulièrement mis au point des anémomètres (qui mesurent la vitesse du vent) et des girouettes (qui mesurent la direction) de plus en plus sophistiqués. Dans cette partie, nous découvrirons les différentes catégories de capteurs qui existent actuellement pour mesurer le vent.

Comme les déplacements d'air verticaux sont en général négligeables par rapport aux déplacements horizontaux, les appareils les plus couramment utilisés pour mesurer le vent fournissent les composantes horizontales mais négligent la composante verticale. Dans cette série d'appareils on peut évoquer l'anémomètre à coupelles présenté en figure 6 , qui possède une vitesse de démarrage de $0,25 \mathrm{~m} / \mathrm{s}$ et qui fonctionne jusqu'à 75 $\mathrm{m} \mathrm{s}-1$ avec une précision de $\pm 0,1 \mathrm{~m} \mathrm{~s}-1$. Pour ce genre d'appareil c'est la rotation des coupelles qui indique la vitesse du vent horizontal. La girouette, présentée sur la même figure, mesure la direction du vent dans le plan horizontal à une résolution de $\pm 2^{\circ}$ à partir d'un seuil de démarrage de $0,6 \mathrm{~m} \mathrm{~s}-1$. Ici c'est la rotation de l'empennage qui donne la direction du vent. Certains capteurs disposent d'une option de système de chauffage intégré qui se déclenche en hiver pour éviter les problèmes d'immobilisation dus au gel. Avec les deux types de capteurs présentés ci-dessous, on mesure séparément la vitesse et la direction du vent et on est obligé d'acquérir deux instruments. C'est pourquoi des constructeurs ont imaginé un type d'appareil qui combine à la fois un anémomètre à hélice et une girouette en un unique élément, que l'on appelle un anémogirouette (figure 7). Ici, c'est l'hélice en rotation qui indique la vitesse et tout le capteur pivote grâce à son profil pour indiquer la direction. La précision pour la vitesse du vent est de $\pm 0,3 \mathrm{~m} / \mathrm{s}$ et de $\pm 3^{\circ}$ pour la direction, pour un seuil de sensibilité de 0,5 à $1 \mathrm{~m} / \mathrm{s}$. 
Fig. 6 : Anémomètre à coupelles (gauche) et girouette (droite)
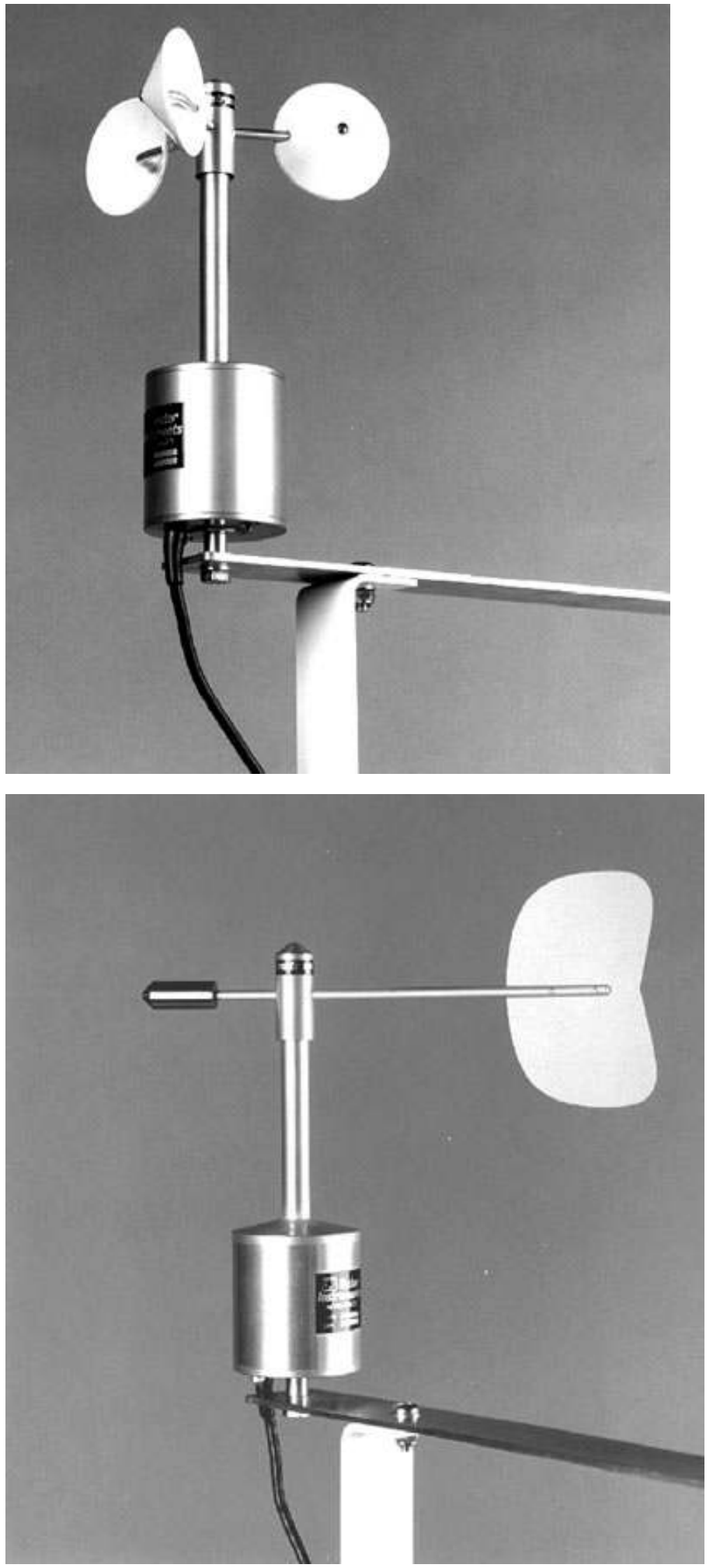

Instruments Vector Instruments, commercialisés par Campbell. Photos : Campbell Scientific. 
Fig. 7 : Anémogirouette

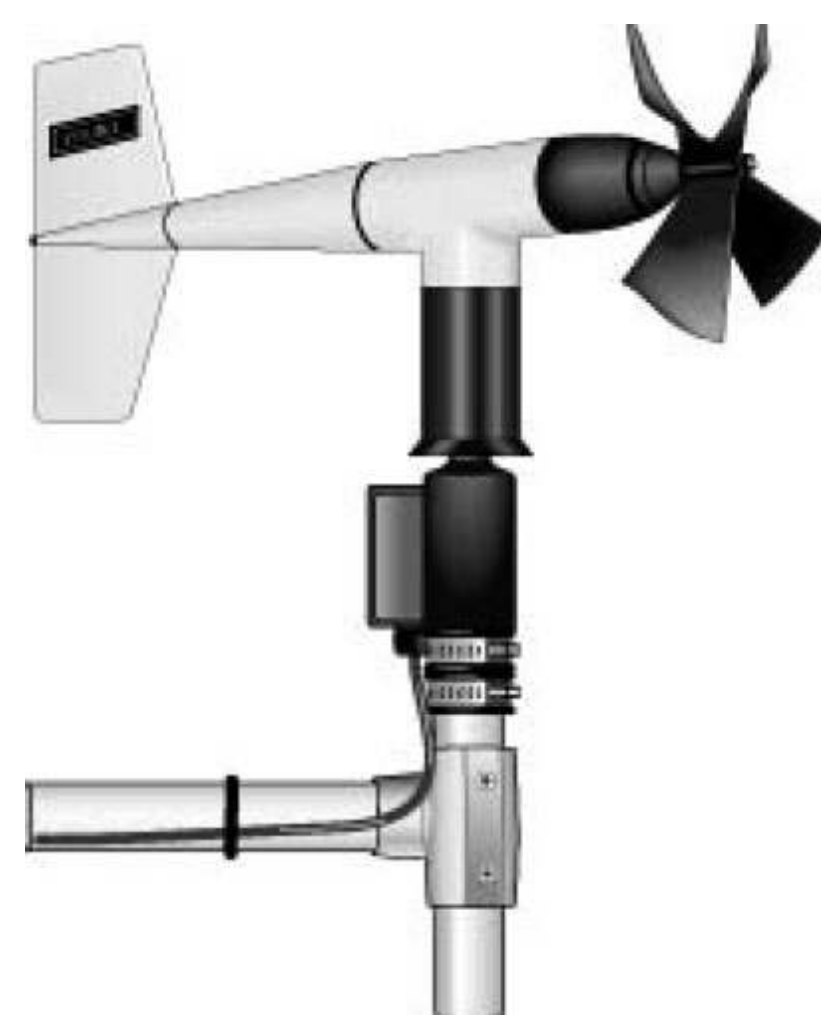

Instrument Young, commercialisé par Campbell. Photo : Campbell Scientific.

8 Si ces appareils simples suffisent dans la majorité des cas, certaines recherches particulières nécessitent la connaissance de la composante verticale du vent. Pour cela on peut utiliser des anémomètres à hélice tridimensionnels (figure 8). Chaque hélice mesure la vitesse du vent selon un axe, et notamment sur la verticale, ce qui permet de reconstituer le vecteur vent en 3D. Les anémomètres soniques possèdent un principe de fonctionnement radicalement différent (figure 9). Ils se passent totalement de parties mécaniques. Des couples de sondes émettent des impulsions ultrasonores selon différents axes. Ces sondes sont utilisées alternativement comme transmetteur et récepteur. L'analyse des transformations subies par les ondes sonores en traversant l'air, donne la possibilité de calculer la vitesse selon ces axes, et d'en déduire la direction du vent dans les 3 dimensions. 
Fig. 8 : Anémomètre tridimensionnel à hélice

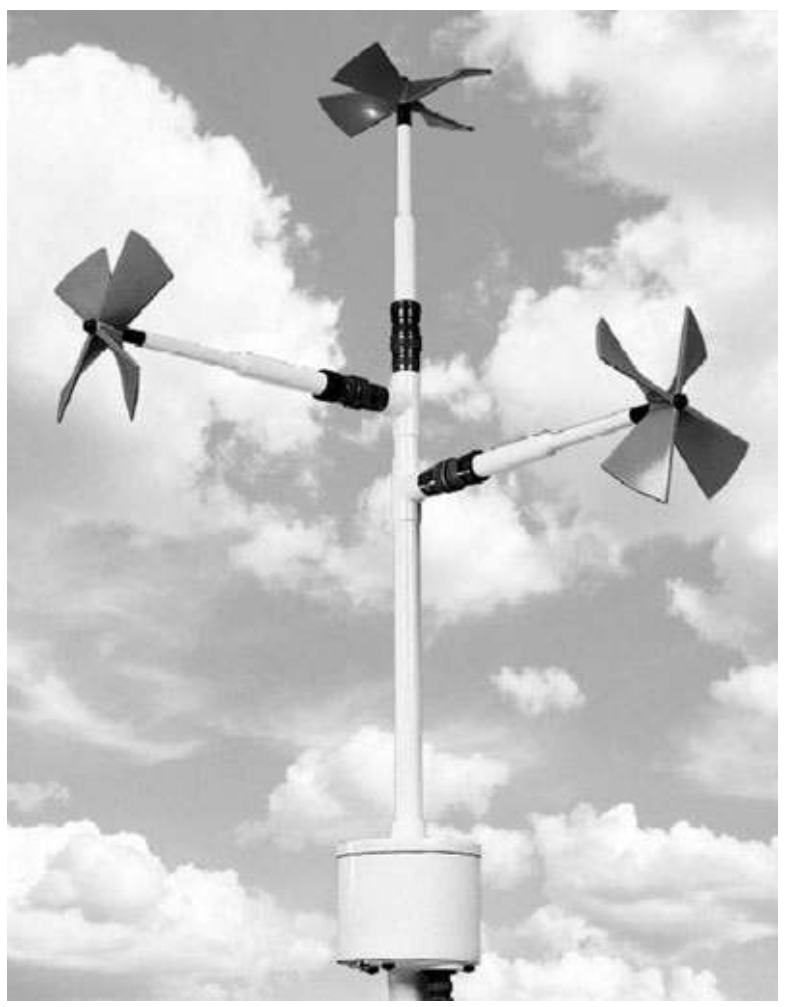

Instrument Gill. Photo : R.M. Young Company.

Fig. 9 : Anémomètre sonique.

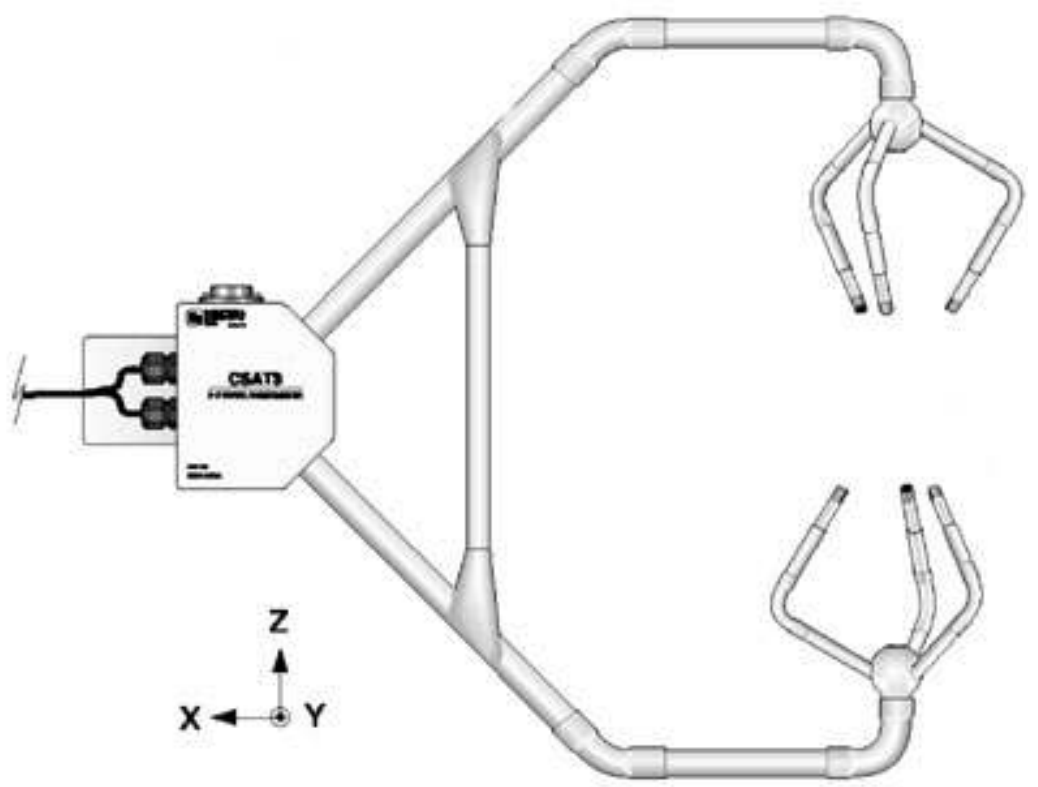

Instrument Campbell. Photo : Campbell Scientific.

9 Les différents capteurs que nous venons d'évoquer équipent les postes au niveau du sol. Mais pour réussir à comprendre certains mécanismes atmosphériques, il est nécessaire de connaître aussi le vent en haute altitude, dans l'atmosphère « libre ». C'est pourquoi, 
pour mesurer le vent loin de la surface, on utilise des ballons captifs, ou des radiosondes. Ce sont des ballons gonflés à l'hélium, et qui sont équipés d'instruments qui transmettent les données au sol par radio.

Les ballons captifs ne peuvent atteindre qu'une altitude limitée puisqu'ils sont reliés au sol par un filin de quelques centaines de mètres. Par contre, ils possèdent l'avantage d'être réutilisables. Les radiosondes, elles, sont capables d'atteindre des altitudes très élevées $(30 \mathrm{~km})$, mais les instruments sont irrécupérables et définitivement perdus, puisque l'engin évolue librement. Ces deux types d'appareils peuvent fournir des profils verticaux de pression, de température, d'humidité et de vent (figure 10).

Fig. 10 : Résultats d'un radiosondage.

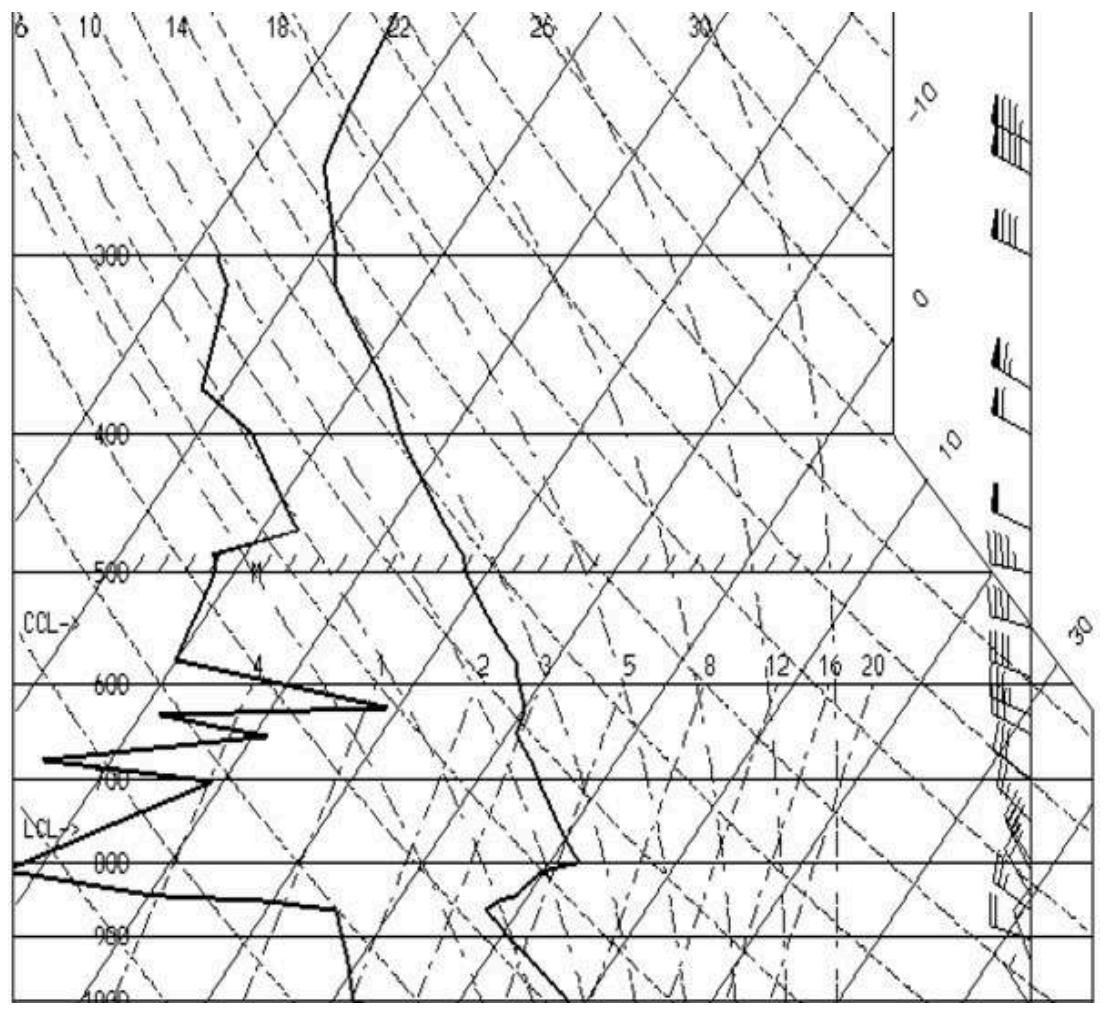

On distingue la courbe du point de rosée, la température, la vitesse et la direction du vent en fonction de la pression (altitude).

Les données des radiosondages sont particulièrement intéressantes, mais les relevés sont effectués à heure fixe 2 fois par jour, à 0 et $12 \mathrm{~h}$ TU, ce qui est insuffisant pour certaines applications qui nécessitent des données en continu. C'est pourquoi, certains appareils permettent de sonder l'atmosphère en continu à partir du sol. Leur principe de fonctionnement repose sur l'émission d'impulsions électromagnétiques qui, lorsqu'elles rencontrent des obstacles ou des interférences, sont réfléchies vers le sol où elles sont réceptionnées et analysées. Ces appareils sont classés en fonction des caractéristiques des impulsions émises. Les RADAR émettent un signal en fréquence radio. Les SODAR utilisent le spectre audible (ils émettent un bourdonnement susceptible de gêner les riverains). Le LIDAR émet un signal dans le spectre visible. Ils utilisent tous le même principe : en connaissant la fréquence du signal émis, sa vitesse, le temps de retour, le décalage de fréquence de l'écho (effet Doppler), on peut 
déterminer la distance de l'interférence et ses propriétés. Ces interférences peuvent être induites par l'humidité, le vent, les variations de températures, ou les aérosols.

Le SODAR par exemple, donne des renseignements sur le vent (vitesse et direction) et la turbulence dans la couche limite planétaire jusqu'à 1000 ou $2000 \mathrm{~m}$. Cet appareil présente donc un intérêt particulier pour les études de trajectoires, pour quantifier la turbulence atmosphérique, pour localiser les zones de cisaillements de vent, connaître les vitesses ascensionnelles... Grâce à ce genre d'appareil on peut ainsi obtenir à intervalles réguliers et très rapprochés, des profils verticaux du vent, comme le montre la figure 11 , ce qui permet une analyse très fine des phénomènes.

Fig. 11 : Profils verticaux horaires du vent obtenus par SODAR

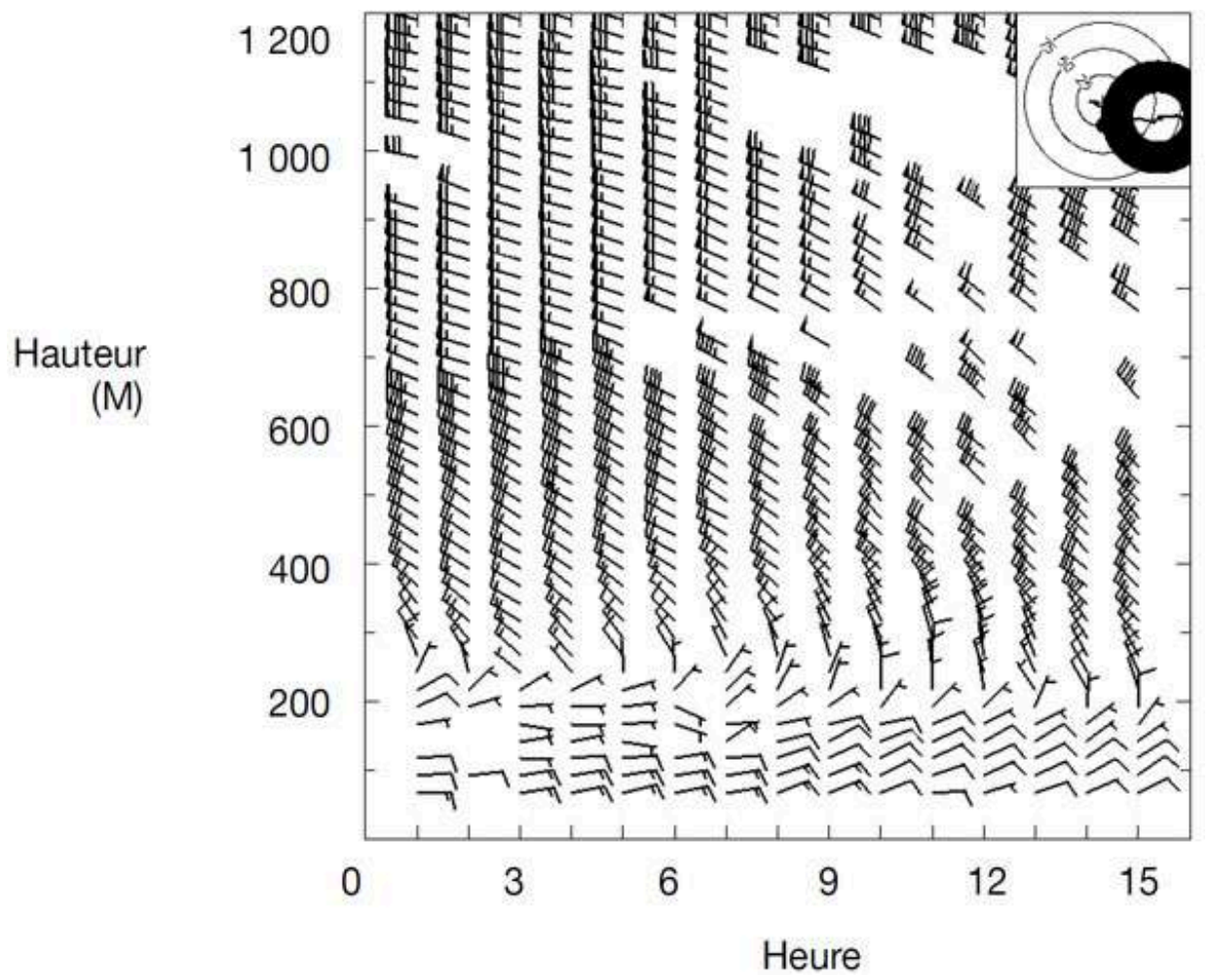

De nouveaux développements sont en cours pour effectuer des mesures à partir d'appareils embarqués sur satellites. Il s'agit de démontrer qu'il est possible de mesurer le vent depuis l'espace par un LIDAR. Des impulsions infrarouges sont émises vers la terre par un laser. A sa traversée de l'atmosphère, une partie du rayonnement est réfléchie vers l'espace par les particules d'air en mouvement, avec un décalage de longueur d'onde, nous donnant ainsi un renseignement sur la circulation atmosphérique. On espère ainsi obtenir une idée plus précise de la circulation atmosphérique dans des secteurs inaccessibles on sous-équipés, ce qui permettra d'étendre la couverture actuelle. Face à ces avantages, l'utilisation de moyens spatiaux ne pourra que s'amplifier dans les années à venir.

\section{L'interprétation des données de vent}

14 Le vent est par essence même, l'exemple typique de variable vectorielle tridimensionnelle qui s'exprime par une orientation (la direction) et un module (la 
vitesse). La direction s'exprime par rapport au Nord et par un angle dont l'unité est le degré (notation ${ }^{\circ}$ ). Les angles évoluent dans le sens des aiguilles d'une montre. Il est important de noter que les vents sont repérés en fonction de leur direction d'origine : un vent d'est, est un vent qui souffle d'est en ouest, et forme un angle de $90^{\circ} \mathrm{par}$ rapport au Nord. Les vitesses s'expriment le plus couramment en mètres par seconde (notation $\mathrm{m} / \mathrm{s}$ ou $\mathrm{m} \mathrm{s}-1$ ) et sont parfois classées selon l'échelle de Beaufort qui s'applique au vent moyen mesuré dix minutes avant l'heure de référence (tableau 1).

Tableau 1 : L'échelle de Beaufort

\begin{tabular}{|c|c|c|c|c|}
\hline $\begin{array}{c}\text { Classes } \\
\text { Beaufort }\end{array}$ & $\begin{array}{c}\text { Types } \\
\text { de vents }\end{array}$ & $\begin{array}{c}\text { Vitesse moyenne } \\
\text { en nœuds }\end{array}$ & $\begin{array}{c}\text { Vitesse moyenne } \\
\text { en } \mathrm{km} / \mathrm{h}\end{array}$ & $\begin{array}{c}\text { Vitesse moyenne } \\
\text { sur } 10 \text { min. (en m/s) }\end{array}$ \\
\hline 0 & Calme & $<1$ & 1 & $<0,27$ \\
\hline 1 & Très légère brise & $1-3$ & $6,27-1,5$ \\
\hline 2 & Légère brise & $4-6$ & $12-19$ & $1,6-3,2$ \\
\hline 3 & Petite brise & $7-10$ & $20-28$ & $3,3-5,4$ \\
\hline 4 & Jolie brise & $11-16$ & $29-38$ & $5,5-7,9$ \\
\hline 5 & Bonne brise & $17-21$ & $39-49$ & $10,8-13,8$ \\
\hline 6 & Vent frais & $22-27$ & $50-61$ & $13,9-17,1$ \\
\hline 7 & Grand frais & $28-33$ & $62-74$ & $17,2-20,7$ \\
\hline 8 & Coup de vent & $34-40$ & $75-88$ & $20,8-24,6$ \\
\hline 9 & Fort coup de vent & $41-47$ & $89-102$ & $24,7-28,5$ \\
\hline 10 & Tempête & $48-55$ & $103-117$ & $28,6-32,6$ \\
\hline 11 & Violente tempête & $56-63$ & $>118$ & $>32,7$ \\
\hline 12 & Ouragan & $>64$ & & 10,7 \\
\hline
\end{tabular}

15 En analysant ce tableau, on remarque notamment que le qualificatif de calme s'accorde pour des vents dont les vitesses moyennes sur 10 minutes sont inférieures à $0,27 \mathrm{~m} / \mathrm{s}$ (1 $\mathrm{km} / \mathrm{h}$ ) et que celui de tempête s'acquiert à partir du moment où le phénomène atteint des vitesses moyennes de l'ordre de $25 \mathrm{~m} / \mathrm{s}(100 \mathrm{~km} / \mathrm{h})$. On peut parler d'ouragan à partir de $32,7 \mathrm{~m} / \mathrm{s}(118 \mathrm{~km} / \mathrm{h})$.

La tempête qui a frappé le sud de la France du 27 au 28 décembre 1999 a généré des vents qui ont bien dépassé en moyenne sur 10 minutes les $118 \mathrm{~km} / \mathrm{h}$ en certains endroits. La valeur la plus élevée a été relevée à la station de Cap Ferret (Gironde) où le vent maximum moyen sur 10 minutes a atteint $133,2 \mathrm{~km} / \mathrm{h}$ le 27 décembre autour de 18 h 23. Aussi ce phénomène peut-il être qualifié d'ouragan. Lors de la tempête qui a frappé le nord de la France le 26 décembre 1999, les vitesses instantanées maximales enregistrées l'ont été lors des rafales à Strasbourg et Nancy $(144 \mathrm{~km} / \mathrm{h})$, à Metz (155 $\mathrm{km} / \mathrm{h})$ et à Colmar $(165 \mathrm{~km} / \mathrm{h})$.

On peut noter que la vitesse maximale mondiale a été atteinte en 1934 au Mont Washington (USA) avec $370 \mathrm{~km} / \mathrm{h}$, et que le record français est détenu par la station du Mont Ventoux en 1967 avec $320 \mathrm{~km} / \mathrm{h}$. Il n'est pas étonnant de constater que ces records ont tous été enregistrés sur les sommets de montagnes très exposés aux vents et non pas dans des plaines ou des vallées, où les vents sont freinés à l'approche du sol, comme nous l'avons évoqué ci-dessus. A titre d'exemple, la figure 12 nous donne les fréquences des vitesses du vent enregistrées pendant 10 ans (période 1987-1997) à la station de recherche météorologique d'Aubure, située sur un sommet vosgien à 1100 mètres. Ces données représentent des moyennes sur 10 minutes. Avec une fréquence de 
plus de $14,7 \%$, ce sont les vents de $5 \mathrm{~m} / \mathrm{s}$ qui sont les plus fréquents, ce qui ferait de ce sommet un éventuel candidat pour un site éolien. La gamme des vitesses s'échelonne de 0 à plus de $29 \mathrm{~m} / \mathrm{s}$, soit plus de $104 \mathrm{~km} / \mathrm{h}$. Malgré la faible durée de la série de mesures, puisqu'il faut 30 ans pour établir une normale climatique fiable, ces données soulignent le caractère tout à fait exceptionnel de l'événement du 26 décembre dont l'intensité n'a pas faibli dans la plaine d'Alsace, alors que cette dernière se trouve pourtant en position d'abri par rapport à la trajectoire du phénomène, puisque située sous le vent de la barrière orographique des Vosges (REKLIP, 1995).

Fig. 12 : Fréquence des vitesses du vent enregistrées de 1987 à 1997 à la station météorologique d'Aubure (1 $100 \mathrm{~m}$, Alsace)

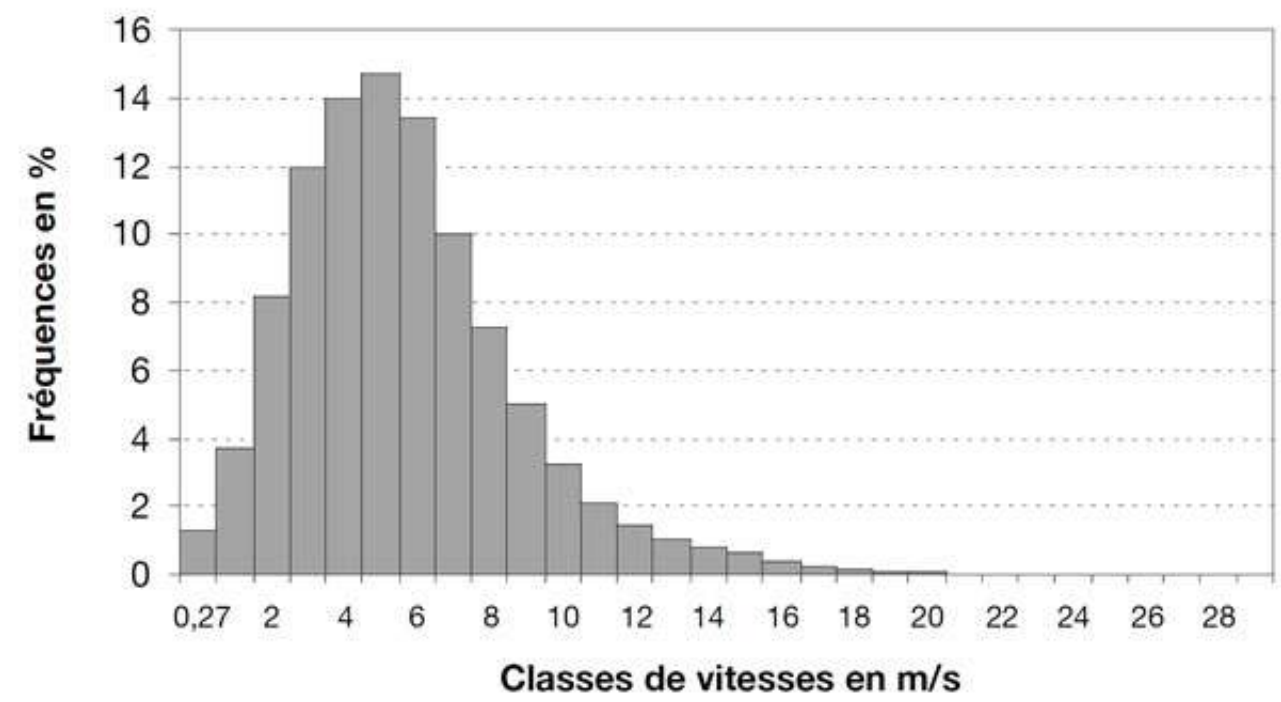

D'après ce qui précède, il est important de noter que la variable vent est particulière dans le sens où l'atmosphère est un fluide qui se déplace de façon irrégulière, chaotique par essence, ce qui entraîne de la turbulence et la formation de rafales tout à fait imprévisibles qui peuvent frapper au hasard. Une rafale est un renforcement brutal et passager du vent. Une rafale peut atteindre une vitesse supérieure de $50 \%$ à celle du vent moyen. C'est donc au cours de rafales, souvent de courte durée, que les vitesses de pointe sont enregistrées et que les dégâts sont le plus souvent occasionnés au niveau du sol. Pour bien comprendre cette notion, on peut se reporter à la figure 13. Sur ce document, qui illustre les vitesses enregistrées sur un sommet vosgien (le Kastelberg, à $1350 \mathrm{~m}$ d'altitude) et sur une période de 3 jours ( 3 au 5 juin 1994), on se rend compte $\mathrm{du}$ caractère essentiellement turbulent $\mathrm{du}$ vent et des rafales, particulièrement bien visibles sur le zoom (en médaillon). Ces rafales ne sont pas représentatives de la vitesse moyenne, puisqu'au moment le plus fort, certaines dépassent les $25 \mathrm{~m} / \mathrm{s}$, alors que la vitesse moyenne à ce moment est de l'ordre de $20 \mathrm{~m} / \mathrm{s}$. Cet événement est qualifié de fort coup de vent. 
Fig. 13 : Vitesse du vent enregistrée du 3 au 5 juin 1994 à la station de recherche du Kastelberg (1 $350 \mathrm{~m}$, Alsace)

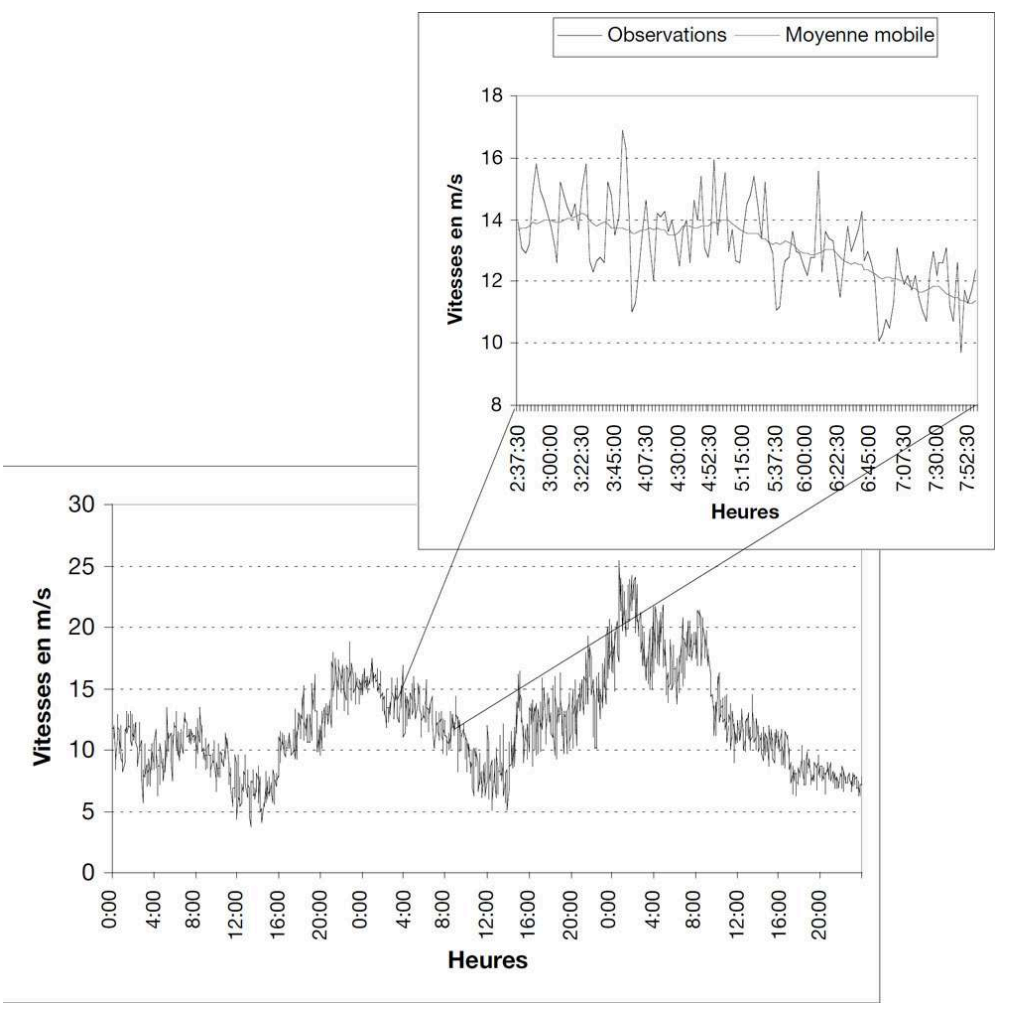

Les données représentent des moyennes obtenues toutes les 2 minutes 30 secondes.

Agrandissement en médaillon et fluctuations par rapport à la moyenne horaire (données lissées).

Sur la période (1987-1997) on observe, sur la rose des vents de la station météorologique d'Aubure (figure 14), que la grande majorité du flux qui balaie les sommets vosgiens provient d'ouest ou de sud-ouest, conformément au principe de circulation des masses d'air qui règne à nos latitudes. La trajectoire de l'événement du 26 décembre est donc conforme au flux dominant sur nos régions. 
Fig. 14 : Fréquences des directions du vent enregistrées de 1987 à 1997 à la station météorologique d'Aubure (1 $100 \mathrm{~m}$, Alsace)

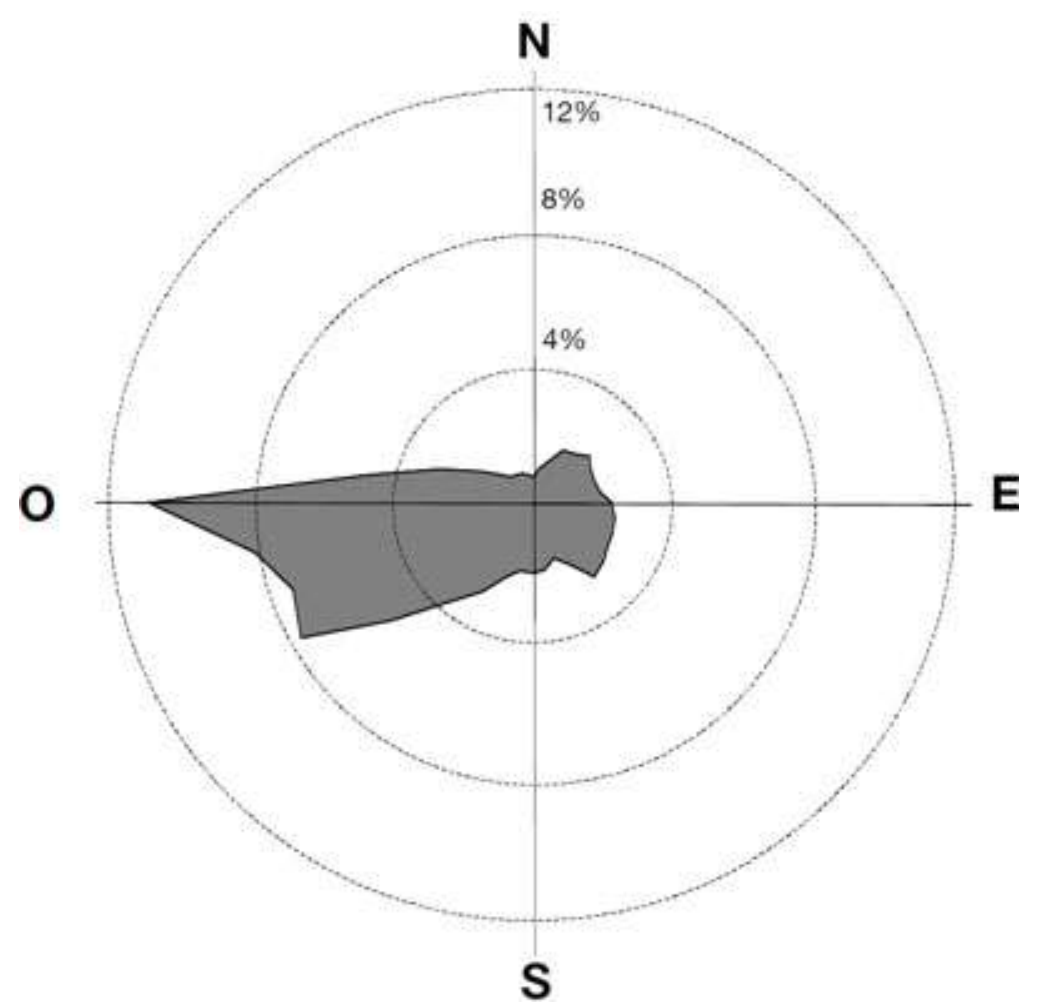

Chaque cercle indique une fréquence de $4 \%$.

\section{Conclusion}

Dans cet article, nous avons voulu présenter une mise au point les moyens techniques qui sont mis en œuvre en météorologie pour mesurer le vent. Ces moyens sont de plus en plus sophistiqués et en perpétuelle évolution du fait des progrès techniques et électroniques. D'autre part, face à l'importance des questions qui lui sont posées, la recherche a sans cesse besoin de nouveaux appareils pour lui permettre de mieux comprendre les phénomènes, voire même pour améliorer la prévision de ces derniers. En cela, les réseaux météorologiques sont encore aujourd'hui irremplaçables puisqu'ils maintiennent l'atmosphère sous étroite surveillance.

\section{BIBLIOGRAPHIE}

Beltrando G. (1998). - Les gelées printanières en champagne viticole. La Météorologie, 21, pp. $30-43$.

Guyot G. (1997). - Climatologie de l'environnement. Masson, 505 p. 
Kastendeuch P.P. et Kaufmann P. (1996). - Classification of summer wind fields over complex terrain. Int. J. Climatol., 17, pp. 521-534.

Kastendeuch P.P., Lacarrère P., Najjar G., Noilhan J., Gassmann F., Paul P. (2000). - Mesoscale simulations of thermodynamic fluxes over complex terrain. Int. J. Climatol., 20, pp. 1249-1264.

Laali A.-R. et Benard M. (1999). - Production d'électricité par énergie éolienne. Situation dans le monde et programme français Eole 2005. La Météorologie, 25, pp. 38-48.

OMM (1996). - Guide des instruments et des méthodes d'observation météorologiques. Sixième édition ; suppléments.

Reklip (1995). - Atlas climatique du Fossé Rhénan Méridional. Editions Coprur.

\section{RÉSUMÉS}

Dans cet article sont présentés les différents moyens qui permettent aux climatologues et aux météorologues de mesurer le vent. Suivent les précautions qui doivent être prises pour installer les appareils et les conventions qui sont utilisées, puis les différents types d'instruments qui sont employés pour mesurer la direction et la vitesse du vent ainsi que leurs limites. Enfin quelques mesures sont interprétées pour bien montrer le caractère exceptionnel des événements de décembre 1999.

In this article the different means available for the climatologists and meteorologists to measure the wind are presented, followed by the precautions and the conventions that must be adopted to install the instruments. The different types of instruments used to measure wind speed and direction and their limitations are considered next. Finally some data showing the exceptional nature of the December 1999 storm are interpreted.

In diesem Beitrag werden die verschiedenen Mittel vorgestellt, mit denen die Klimatologen und Meteorologen den Wind messen: Die Mass-nahmen zur Einrichtung der Apparate, die angewandten Methoden und die verschiedenen Instrumenttypen zur Messung von Richtung, Geschwindigkeit und Grenzen der Winde. Schliesslich werden einige Messungen interpretiert, um den aussergewöhnlichen Charakter der Ereignisse vom Dezember 1999 zu zeigen.

\section{INDEX}

Schlüsselwörter : Anemometer, Instrumente, meteorologisches Messnetz, Wind, Windmesser (Wetterfahne)

Keywords : anemometer, instruments, meteorological network, wind, wind vane

Mots-clés : anémomètre, girouette, instruments, réseau de mesures météorologique, vent

\section{AUTEUR}

\section{PIERRE-PHILIPPE KASTENDEUCH}

Université Louis Pasteur Strasbourg I - Faculté de Géographie et d'Aménagement - CEREG - 3, rue de l'Argonne 67083 Strasbourg Cedex 